\title{
In Vivo PET Imaging Demonstrates Diminished Microglial Activation After Fingolimod Treatment in an Animal Model of Multiple Sclerosis
}

\author{
Laura Airas $^{1}$, Alex M. Dickens², Petri Elo², Päivi Marjamäki², Jarkko Johansson ${ }^{3}$, Olli Eskola ${ }^{4}$, Paul A. Jones ${ }^{5}$, \\ William Trigg ${ }^{5}$, Olof Solin ${ }^{4}$, Merja Haaparanta-Solin ${ }^{2}$, Daniel C. Anthony ${ }^{6}$, and Juha Rinne ${ }^{3}$ \\ ${ }^{1}$ Division of Clinical Neurosciences, Turku University Hospital, Turku, Finland; ${ }^{2}$ Medicity/Preclinical Imaging, Turku PET \\ Centre, University of Turku, Turku, Finland; ${ }^{3}$ Clinical Neurology, Turku PET Centre, University of Turku, Turku, Finland; \\ ${ }^{4}$ Radiopharmaceutical Chemistry Laboratory, Turku PET Centre, University of Turku, Turku, Finland; ${ }^{5}$ GE Healthcare Ltd., The \\ Grove Centre, Amersham, United Kingdom; and ${ }^{6}$ Department of Pharmacology, University of Oxford, Oxford, United Kingdom
}

There is a great need for the monitoring of microglial activation surrounding multiple sclerosis lesions because the activation of microglia is thought to drive widespread neuronal damage. Recently, second-generation PET radioligands that can reveal the extent of microglial activation by quantifying the increased expression of the $18-\mathrm{kDa}$ translocator protein have been developed. Here, we investigate whether PET imaging can be used to demonstrate the reduction in microglial activation surrounding a chronic focal multiple sclerosis (MS)-like lesion after treatment with fingolimod, an established MS therapy. Methods: Chronic focal experimental autoimmune encephalitis (EAE)-like lesions were induced in Lewis rats $(n=24)$ via stereotactic intrastriatal injection of heat-killed bacillus Calmette-Guérin (BCG) and subsequent activation using an intradermal injection of BCG in complete Freund adjuvant. This process resulted in a delayed-type hypersensitivity (DTH)-like EAE lesion. The extent of neuroinflammation surrounding the lesion was measured using ${ }^{18} \mathrm{~F}-\mathrm{GE} 180$ as a PET radioligand. The imaging was performed before and after treatment with fingolimod $(0.3 \mathrm{mg} / \mathrm{kg} / \mathrm{d}$ by mouth, $28 \mathrm{~d}$ ) or vehicle as a control. In addition to imaging, autoradiography and immunohistochemistry experiments were performed to verify the in vivo results. Results: The chronic DTH EAE lesion led to increased ligand binding in the ipsilateral, compared with contralateral, hemisphere when PET imaging was performed with the translocator protein-binding radioligand ${ }^{18} \mathrm{~F}-\mathrm{GE} 180$. Treatment with fingolimod led to a highly significant reduction in the binding potential, which could be demonstrated using both in vivo and ex vivo imaging (fingolimod vs. vehicle treatment, $P<0.0001$ ). The area of increased ${ }^{18} \mathrm{~F}-\mathrm{GE} 180$ signal mapped closely to the area of activated microglial cells detected by immunohistochemistry. Conclusion: PET imaging, unlike MR imaging, can be used to visualize the microglial activation surrounding a chronic DTH EAE lesion. Importantly, the treatment effect of fingolimod can be monitored in vivo by measuring the degree of microglial activation surrounding the chronic DTH EAE lesion. This work gives promise for the introduction of new outcome measures applicable in treatment studies of progressive MS.

Received Oct. 13, 2014; revision accepted Dec. 8, 2014.

For correspondence or reprints contact: Laura Airas, Division of Clinical Neurosciences, Turku University Hospital, Kiinamyllynkatu 4-8, 20521 Turku, Finland.

E-mail: laura.airas@utu.fi

Published online Jan. 8, 2015.

COPYRIGHT (C 2015 by the Society of Nuclear Medicine and Molecular Imaging, Inc.
Key Words: multiple sclerosis; PET imaging; TSPO; fingolimod; neuroinflammation

J Nucl Med 2015; 56:305-310

DOI: 10.2967/jnumed.114.149955

\section{$\mathbf{E}$}

ndpoints for development of new therapies for multiple sclerosis (MS) have conventionally been the suppression of relapses, reduction in the number of gadolinium-enhancing or new or enlarging T2 lesions on MR imaging, and evaluation of disability using the expanded disability status scale (1). However, it is increasingly clear that evaluation of the diffuse inflammation associated with progressive MS and follow-up of the development of neurodegeneration need more accurate and sensitive imaging methods than can be achieved using MR imaging (2). Recent work suggests that neuronal damage in the MS brain is associated with microglial activation and is more widespread than previously envisaged. In particular, axonal damage occurs not only in MS lesions but also within the normalappearing white matter and spatially correlates to regions of activated microglia (3). In addition, it is clear that current MR imaging techniques are insensitive to the detection of pathologies associated with progression of MS (4). There is thus an urgent need to develop novel imaging methods for the detection and monitoring of these processes. Methods that can accurately report on extralesional pathologic processes may then be used to establish what therapies, if any, are able to modify this more widespread, extralesional damage (5).

It has been long recognized that microglial activation is associated with the increased expression of the $18-\mathrm{kDa}$ translocator protein (TSPO), previously known as the peripheral benzodiazepine receptor (5). Thus, an increase in expression of TSPO has been proposed as a surrogate marker for neuroinflammation (6). The increase in expression of TSPO can be visualized using PET and an appropriate radiotracer such as ${ }^{11} \mathrm{C}-\mathrm{PK} 11195$ (7-9). Recent years have seen the development of so-called second-generation TSPO radioligands, which have a better signal-to-noise ratio and other more favorable physicochemical characteristics, compared with the ${ }^{11} \mathrm{C}-\mathrm{PK} 11195$ ligand $(10)$. One such radioligand in development is ${ }^{18} \mathrm{~F}-\mathrm{GE} 180(11,12)$.

Fingolimod (FTY720; Gilenya) was the first oral therapy to be approved for treatment of MS (13). Fingolimod is phosphorylated 
in vivo to fingolimod phosphate, which is a broad sphingosine 1-phosphate receptor (S1PR) antagonist and binds to all S1PR apart from the sphingosine 2-phosphate receptor (14). The primary mechanism of action of fingolimod in MS therapy is thought to be modulation of S1PR expression on T cells; fingolimod blocks the T cells from exiting lymph nodes and thus reduces the $\mathrm{T}$ cell-mediated autoimmune reaction in the central nervous system (CNS) (15). However, fingolimod also readily enters the CNS in which it can bind to neurons, oligodendrocyte progenitor cells, astrocytes, and microglia $(13,16)$. It has been demonstrated in vitro that fingolimod can suppress the activation of microglia (17). The primary aim of this study was to evaluate whether fingolimod reduces microglial activation in an MS animal model when administered to the animals and whether ${ }^{18} \mathrm{~F}-\mathrm{GE} 180$ can be used to monitor the impact of treatment on microglial activation in vivo. To study the effect of fingolimod, we used a delayed-type hypersensitivity (DTH) model of a pattern I-type MS lesion characterized by a $\mathrm{T}$ cell- and macrophagedominated lesion $(18,19)$. Using this animal model, we aimed to investigate the microglial activation within the lesion area and in the area surrounding the lesion. The formation of stratified lesions in terms of spatial distribution and kinetics distinguishes this model from other types of experimental autoimmune encephalomyelitis (EAE), in which lesion presentation is random and variable and hence not quantifiable (20). Regarding lesion pathology, the DTH lesion closely resembles lesions seen in MS, including blood-brain barrier (BBB) breakdown at the height of the disease, demyelination, $\mathrm{T}$ cell and macrophage infiltration, and widespread microglial activation (18).

\section{MATERIALS AND METHODS}

\section{Animals}

All animal experiments were performed with the required ethical approval from the Southern Finland ethics board (application no. ESAVI/6360/04.10.03/2011). Male Lewis rats (50-100 g, $n=18$ ) were obtained from Charles River and allowed to acclimatize for $1 \mathrm{wk}$ before the start of any experiments. All animals were housed in accordance with the Amsterdam protocol for animal experiments (21); food and water were available ad libitum.

\section{Intrastriatal Injection of Bacillus Calmette-Guérin (BCG)}

Before stereotactic surgery the animals were anesthetized using isoflurane (4\%) Baxter Medical $\mathrm{AB}$ ) in air. Anesthesia was maintained with $2.5 \%$ isoflurane, and body temperature was maintained using a heating blanket. The rats were placed onto a stereotactic frame (Kopf Instruments), and the skull was exposed. The brain of the animal was exposed via a hole drilled though the skull. Heatkilled BCG $\left(1 \times 10^{5}\right.$ cells in $2 \mu \mathrm{L}$ of phosphate-buffered saline $)$ was injected into the left striatum using a Hamilton syringe $(10 \mu \mathrm{L}$; Sigma Aldrich). The acute inflammatory response to the intracranial injection of BCG resolves rapidly. The BCG remains sequestered behind the $\mathrm{BBB}$ and will escape recognition by the immune system (22).

\section{Activation of Lesion}

Twenty-eight days after the intracerebral BCG injection, the lesions were activated by an intradermal injection of BCG (1.5 mg; Difco) in an emulsion of complete Freund adjuvant (100 $\mu \mathrm{L}$; Sigma Aldrich) mixed with phosphate-buffered saline $(100 \mu \mathrm{L})$. This peripheral sensitization of the immune system provokes an immune cell-mediated attack toward the intracranial BCG deposit, resulting in the formation of focal DTH lesions and bystander damage in the CNS (22).

\section{Experimental Design}

After the peripheral activation of the lesion, the DTH EAE lesions were allowed to develop until day 127 to generate large chronic lesions and the animals were then treated for $28 \mathrm{~d}$ with either fingolimod $(n=7)$ or vehicle $(n=7$; Fig. 1$)$. The fingolimod treatment was given daily $(0.3 \mathrm{mg} / \mathrm{kg}$ in $0.5 \mathrm{~mL}$ of water). The control group was given water $(0.5 \mathrm{~mL})$ as a vehicle control. The animals were dosed via oral gavage to ensure accurate dosing. In this model, the acute inflammation subsides after day 20 of activation of the lesion, and the BBB damage subsides (23). Thus, at day 127 the lesion clearly represents a well-developed chronic MS lesion. PET imaging was performed immediately before initiation of treatment and at the end of the treatment period.

\section{Radiotracer Production}

${ }^{18} \mathrm{~F}-\mathrm{GE} 180$ was synthesized using a previously published method (12). Briefly, ${ }^{18} \mathrm{~F}-\mathrm{F}^{-}$was generated by proton irradiation of ${ }^{18} \mathrm{O}-$ enriched $\mathrm{H}_{2} \mathrm{O}$ (97\% enrichment). After suitable workup, the ${ }^{18} \mathrm{~F}_{-} \mathrm{F}^{-}$ anion was then reacted with the precursor molecule (GE Healthcare), resulting in the formation of ${ }^{18} \mathrm{~F}-\mathrm{GE} 180$. Finally, the tracer was purified with semipreparative high-performance liquid chromatography and formulated for injection (12).

\section{In Vivo Imaging}

In vivo imaging was performed using an Inveon multimodality PET/CT (Siemens Medical Solutions, USA) small-animal scanner with a $1.2 \mathrm{~mm}^{3}$ resolution. The device generates images with 159 transaxial slices with a $10-\mathrm{cm}$ transaxial field of view and a $12.7-\mathrm{cm}$ axial field of view. A CT scan was obtained to correct for signal attenuation in the PET scan and provide anatomic references. Emission scans $(40 \mathrm{~min}$ ) were acquired for these studies. The data were acquired in list-mode with an emission window from 350 to $650 \mathrm{MeV}$. The scans were initiated immediately after the intravenous injection of ${ }^{18} \mathrm{~F}-\mathrm{GE} 180$. For the baseline scan, the amount of radioactivity given to animals in the control group was $49.0 \pm 3.1 \mathrm{MBq}$ (mean $\pm \mathrm{SD}$ ), and in the fingolimod-treated group it was $46.5 \pm 6.3 \mathrm{MBq}$. In the second scan, the amounts were $46.6 \pm 5.2$ and $45.7 \pm 8.6 \mathrm{MBq}$, respectively.

\section{Digital Autoradiography}

Rats were killed for autoradiography analysis $(n=3)$ after the 40-min in vivo PET imaging. The animals were killed $45 \mathrm{~min}$ after tracer injection, the anesthetic was increased to a terminal level (5\% in air), and the thoracic cavity was opened. Blood was collected via a cardiac puncture, and the animals were transcardially perfused with heparinized saline $(20 \mathrm{~mL})$. The brains were removed, frozen, and sectioned $(20 \mu \mathrm{m})$ in a cryostat (Leica). Coronal sections were taken from different brain regions (olfactory bulbs, striatum, cerebellum) and exposed to a phosphor imaging plate (BAS-TR2025; Fuji Photo Film Co., Ltd.) for 2 half-lives of the radiotracer $(220 \mathrm{~min})$. The plates

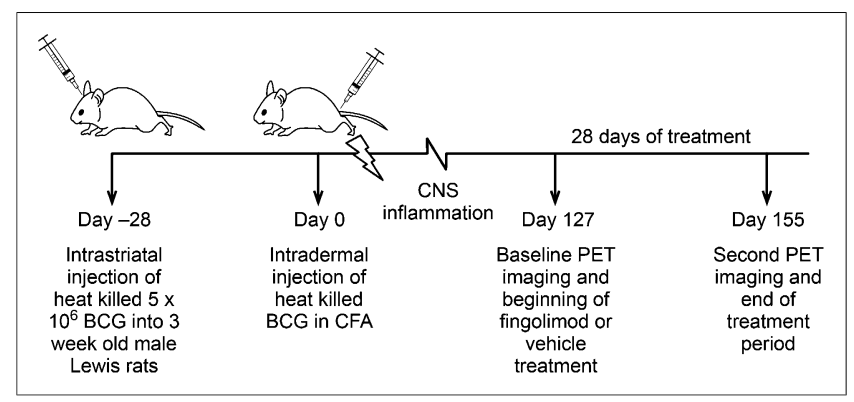

FIGURE 1. Experimental design showing timeline of procedures. 


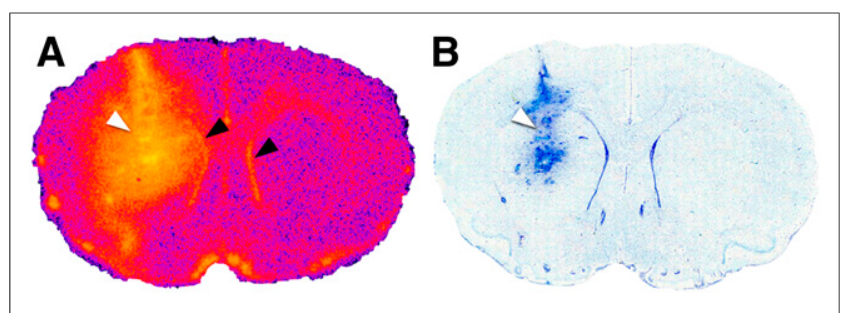

FIGURE 2. (A) Example of digital autoradiography image of coronal slice from striatum at beginning of treatment period. White arrowheads mark site of injection, and black arrowheads indicate radioligand uptake in ventricles. Area of high binding of ${ }^{18} \mathrm{~F}-\mathrm{GE} 180$ is indicated in yellow (lesion hemisphere), and low binding of ${ }^{18} \mathrm{~F}-\mathrm{GE} 180$ is indicated in purple (control hemisphere). (B) Corresponding section stained with cresyl violet, demonstrating extent of lesion in left striatum (white arrowhead). ${ }^{18} \mathrm{~F}-\mathrm{GE} 180$ binds not only to lesion core, but also to surrounding inflamed area as well.

were then interpreted in a phosphor imaging plate interpreter (BAS5000 [Fuji Photo Film Co., Ltd.]; $25 \mathrm{~mm}^{2}$ resolution). Finally, the sections were frozen.

Regions of interest (ROIs) were drawn in the left and right striatal and cortical areas on the images obtained from autoradiography. The digital autoradiography images were analyzed for count densities (photostimulated luminescence per unit area, PSL $/ \mathrm{mm}^{2}$ ) with a computerized image analysis program (Aida 2D densitometry program; Raytest Isotopenmessgeräte $\mathrm{GmbH}$ ). ROIs were drawn over the left and right hemisphere at the level of the striatum and cerebellum. To quantify the binding potential $\left(\mathrm{B}_{\mathrm{ND}}\right)$ of the radioligand, the following calculation was performed:

$$
\mathrm{B}_{\mathrm{nd}}=\left(\mathrm{ROI}_{(\text {lesion })}-\mathrm{ROI}_{(\text {contralateral })}\right) / \mathrm{ROI}_{(\text {contralateral })}
$$

The result of the equation was then averaged across the whole striatum to get an overall binding ratio for each injected animal. This binding potential was then normalized to the total lesion volume estimated from the cresyl staining (below).

In addition to the sections used in the autoradiography, some sections were directly cut from the striatum and frozen for immunohistochemistry analysis. The fresh sections were post-fixed with periodate-lysine-paraformaldehyde as previously described (24). Endogenous peroxide activity was quenched by placing the sections in a $1 \% \mathrm{H}_{2} \mathrm{O}_{2}$ solution in $\mathrm{MeOH}$ for $20 \mathrm{~min}$. The sections were then incubated with horse serum for $1 \mathrm{~h}$ before the addition of the primary antibody (polyclonal goat anti-IBA-1; Abcam) for incubation overnight at $4^{\circ} \mathrm{C}$. The sections were then incubated with a biotinylated secondary antibody (Vector labs). The signal was amplified using an avidin biotin system (ABC kit; Vector Labs) and then developed using 3,3-diaminobenzidine. The sections were finally counterstained using cresyl violet. Serial sections were stained with the Bielschowsky silver impregnation method to demonstrate neuronal processes according to the standard Bancroft and Stevens protocol.

The sections that had been used for autoradiography were stained only with cresyl violet because this tissue was not suitable for immunohistochemistry. This staining allowed quantification of the lesion volume for the autoradiography analysis.

\section{In Vivo Data Analysis}

The data were reconstructed using the ordered-subsets expectation maximization algorithm in 3 dimensions plus fastMAP (fast maximum a posteriori) iterative reconstruction protocols in the Inveon acquisition software (Siemens Medical Solutions, USA). All datasets were aligned to a standard rat template (Sciffer, PMOD) using the rigid matching tool within PMOD, version 3.4 (PMOD Technologies Ltd.). The images were then evaluated by drawing a spheric ROI in the injected striatum. This ROI was then mirrored in the contralateral hemisphere to act as a reference region. ROI analysis and segmentation were performed in PMOD. The time-activity curve (TAC) from the volume of interest drawn in the lesioned hemisphere was subtracted from the time-activity curve from the contralateral hemisphere to examine the point of maximal binding (25-50 $\mathrm{min}$ ) during the imaging time frame. The boundto-free ratio was then calculated over the period of maximal binding, as described by Farde et al. (25). Briefly, the area under the curve was calculated by integrating between the defined time limits during the maximal binding period for both the lesioned time-activity curve and the contralateral time-activity curve. To get the bound-to-free ratio, the following calculation was performed:

$$
\mathrm{B}_{\mathrm{nd}}=\left(\mathrm{TAC}_{(\text {lesion })}-\mathrm{TAC}_{(\text {contralateral })}\right) / \mathrm{TAC}_{(\text {contralateral })}
$$

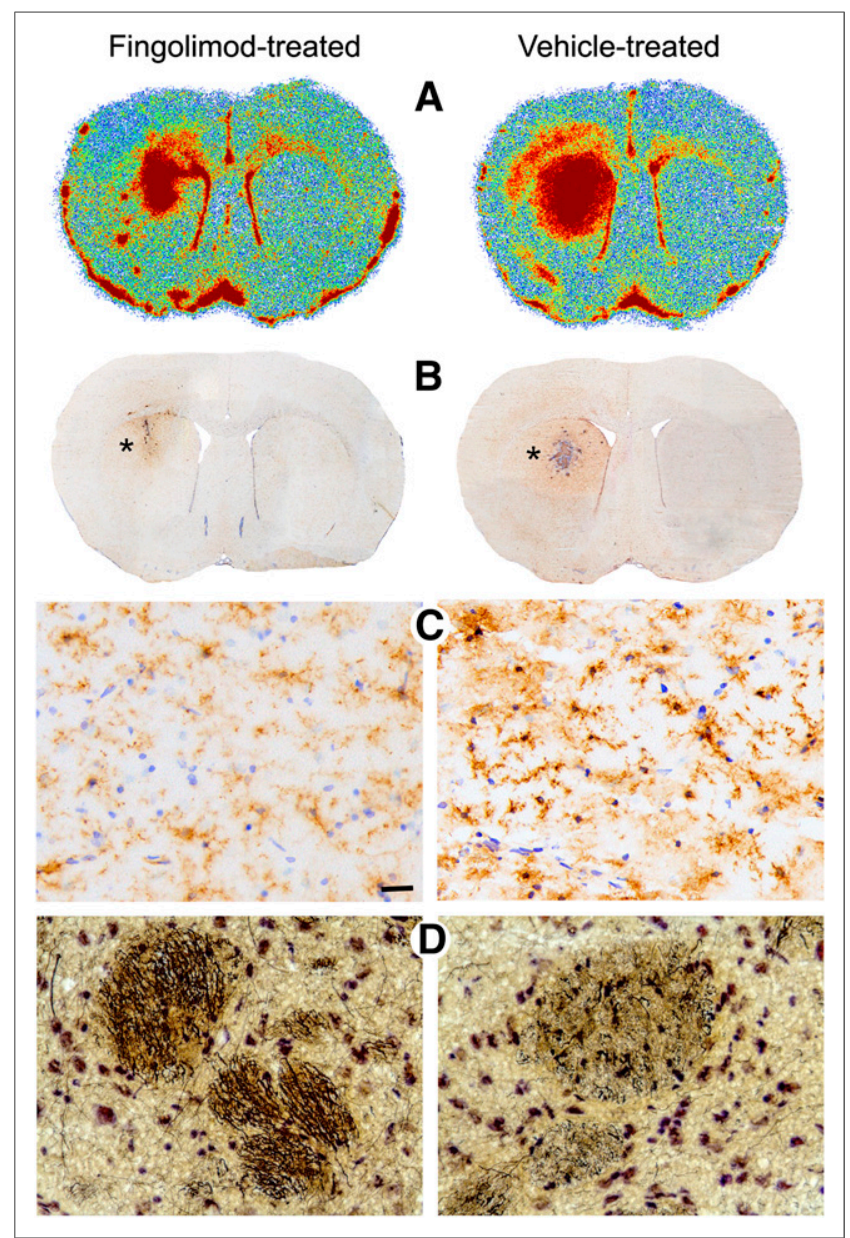

FIGURE 3. Reduced ${ }^{18} \mathrm{~F}-\mathrm{GE} 180$ ex vivo binding after fingolimod treatment is associated with reduced microglial activation. (A) Representative coronal striatal autoradiography images demonstrating area of ${ }^{18} \mathrm{~F}-\mathrm{GE} 180$ binding after treatment with fingolimod or vehicle. (B) Representative wholebrain photomicrographs demonstrating immunohistochemical staining of activated microglia (anti-IBA-1) in fingolimod-treated and vehicle-treated animals. (C) High-powered photomicrographs with anti-IBA-1 staining taken from border of lesion (marked with * in B). (D). In fingolimod-treated animals, silver staining shows normal-appearing axons at site of * in B (left), whereas in vehicle-treated animals axons are dystrophic and reduced in density at corresponding site (right). High power scale bar, $20 \mu \mathrm{m}$. 


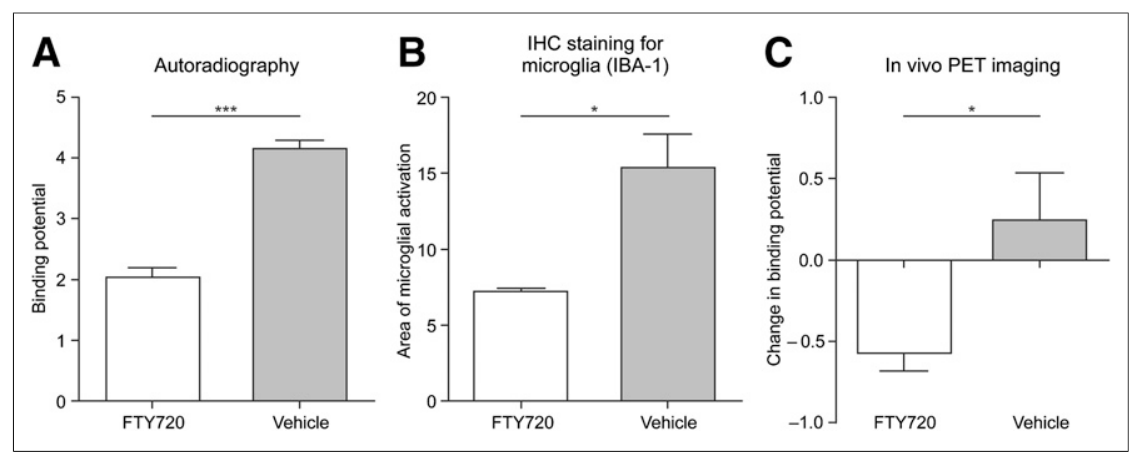

FIGURE 4. Microglial activation surrounding chronic focal EAE lesion is reduced after fingolimod treatment. (A) Autoradiography using ${ }^{18} \mathrm{~F}-\mathrm{GE} 180$. Quantification of radioligand binding after fingolimod treatment (fingolimod, $n=3$, vehicle $n=3$ ) is shown. ${ }^{\star \star \star} P<0.0001$. (B) Immunohistochemical staining using anti-IBA-1 monoclonal antibody. Quantification of area of microglial activation, normalized to lesion area, is shown. ${ }^{*} P<0.05$. (C) In vivo PET using ${ }^{18} \mathrm{~F}-\mathrm{GE} 180$. Quantification of change in binding potential after treatment (fingolimod, $n=5$, white bar; vehicle, $n=3$, gray bar). ${ }^{*} P=0.0206$. $\mathrm{IHC}=$ immunohistochemistry.

\section{Pixelwise Modeling of In Vivo Images}

The images were reconstructed into 3 dimensions using the in-built tool within PMOD, and then auto segmentation of the lesions was performed using the threshold tool and placing a seed point in the lesion area.

\section{Statistical Analysis}

All statistical analysis was performed using Prism (5.01; Graph Pad Software Inc.). A result was considered significant if $P$ was less than 0.05. Images were coregistered using Photoshop (CS4; Adobe) to resize and overlay the images.

\section{RESULTS}

\section{Fingolimod-Reduced Microglial Activation and Ex Vivo ${ }^{18} \mathrm{~F}$-GE180 Binding in Chronic DTH EAE Lesion}

The chronic focal EAE lesion produced by intrastriatal injection of BCG and subsequent peripheral activation caused a large inflammatory lesion in the ipsilateral hemisphere, which was well visualized after $127 \mathrm{~d}$ of lesion induction. This late lesion had a wide area of microglial activation depicted by increased uptake of ${ }^{18} \mathrm{~F}-\mathrm{GE} 180$ in the ipsilateral hemisphere (Fig. 2A) and a hypercellular core visualized by cresyl violet staining (Fig. 2B). No inflammation was observed in the contralateral hemisphere (Fig. 2). The increased ipsilateral microglial activation was not solely limited to the area of hypercellularity but continued into the normal-appearing brain tissue surrounding the lesion and was demonstrated by an increase in perilesional ${ }^{18} \mathrm{~F}-\mathrm{GE} 180$ binding (Fig. 2). Treatment of the animals with fingolimod for $28 \mathrm{~d}$ resulted in a clear reduction in the binding of ${ }^{18} \mathrm{~F}-\mathrm{GE} 180$ when compared with vehicle-treated animals and evaluated by ex vivo autoradiography (Fig. 3A). Quantification of the binding of the radiotracer revealed a significant reduction in the binding potential of ${ }^{18} \mathrm{~F}-\mathrm{GE} 180(P<0.0001)$ after treatment with fingolimod (Fig. 4A).

\section{Evaluation of Microglial Activation and Axonal Integrity in Chronic Focal EAE Using Immunohistochemistry}

The area of increased uptake of the radiotracer corresponded to the area of activated microglia observed by immunohistochemistry (Figs. 3A-3C). Fingolimod treatment prevented lesion expansion, demonstrated by a diminished area of microglial activation surrounding the lesion in immunohistochemical staining (Fig. 4B;
$7.22 \pm 3.40$ arbitrary units [AUs] for fingolimod treatment vs. $15.35 \pm 2.18$ AUs for vehicle treatment, $P=0.0264)$. Closer examination of the appearance of the IBA1 -stained microglia revealed that the cellular processes were more elongated in the fingolimod-treated animals at equivalent extralesional sites, compared with the vehicle-treated animals (Fig. 3C). Silver staining revealed the presence of dystrophic axons surrounding the lesions. At equivalent sites, the silver stain demonstrated axon sparing in the fingolimod-treated animals, compared with vehicle-treated animals (Fig. 3D).

\section{Effect of Fingolimod on Microglial Activation in Chronic Focal EAE Evaluated Using in Vivo TSPO PET Imaging}

From the in vivo PET images it was possible to observe binding of the ${ }^{18} \mathrm{~F}$ GE180 radioligand in the ipsilateral but not contralateral striatum (Fig. 5). When comparing a pretreatment scan with a posttreatment scan, a decrease in the size of the volume of ${ }^{18} \mathrm{~F}-\mathrm{GE} 180$ binding after treatment with fingolimod was observed (Fig. 5). A similar reduction was not observed in the vehicle-treated group; in fact the lesion actually increased in size (Fig. 5B). Quantification of the PET data revealed a significant reduction in the $\mathrm{B}_{\mathrm{nd}}$ in the fingolimodtreated group $(-0.634 \pm 141 \mathrm{AUs})$, compared with the vehicletreated group $(0.266 \pm 0.281$ AUs; $P=0.021)$ (Fig. 4C).

\section{DISCUSSION}

In this study, we have demonstrated for the first time, to our knowledge, that in vivo PET imaging using a TSPO-binding radioligand can be used to quantify microglial activation in a chronic focal EAE model in rats. Importantly, fingolimod treatment of the animals resulted in diminished microglial activation, demonstrable as a reduction both in lesion volume and in binding potential in in vivo PET. Furthermore, this reduction in TSPO binding was observed in autoradiography and could be attributed to reduction



FIGURE 5. In vivo imaging demonstrates reduction in ${ }^{18} \mathrm{~F}-\mathrm{GE} 180$ binding after treatment with fingolimod. (A) Representative coronal slices of in vivo PET images from fingolimod-treated animal or vehicle-treated animal. Top row shows images obtained from animal before treatment, and bottom row shows images after treatment. (B) Average 3D reconstructions of PET images before (top) and after (bottom) treatment with either fingolimod ( $n=5)$ or vehicle $(n=3)$. Red areas indicate increased radiotracer uptake in region of lesion. Blue areas represent ventricles. 
in the activation of the microglia, confirmed by anti-IBA-1 immunostaining. Importantly, earlier extensive work has validated that the expression of TSPO is localized in activated microglial cells and macrophages and to some degree also in astrocytes in abnormal brain both in humans and in rodents $(6,26)$.

Fingolimod efficacy in the treatment of MS has been well demonstrated (27). The impact of fingolimod on the immune system is immunomodulatory and dependent on the retention of lymphocytes within the lymph nodes (28). Because of its lipophilicity, fingolimod crosses the $\mathrm{BBB}$, and pharmacologically relevant concentrations are found in the brain and spinal cord after oral administration (16). There is good evidence from MR imaging that fingolimod reduces brain atrophy development, compared with placebo and interferon- $\beta$ therapy, an observation suggestive of the neuroprotective action of this drug (27). S1PRs are expressed on all CNS cell types, and binding of fingolimod phosphate to these results in intracellular signaling and potentially beneficial outcome effects in terms of preventing progression of MS (29).

Microglial activation is critical in MS pathogenesis. In secondary progressive MS, there is increased microglial activation and accumulating neurodegeneration throughout the white matter and gray matter (30). The observed correlation between diffuse inflammation and axonal injury suggests that inflammation likely contributes to neurodegeneration and disease progression, and thus therapeutic targeting of microglial activation might also reduce the axonal injury (30-32). The present work using the chronic focal EAE model mimicking progressive MS and our earlier human PET imaging study on secondary progressive MS patients (9) together demonstrate that the evaluation of microglial activation using TSPO-binding PET ligands allows assessment of the diffuse brain inflammation and perilesional activity in progressive MS and animal models of the progressive disease in vivo. Importantly, our present work also demonstrates the close correlation between axonal damage and microglial activation.

MS is traditionally seen as an inflammatory autoimmune disease, but also neurodegeneration is present from early on. A plethora of medications has emerged in recent years for treatment of relapsingremitting MS, but there is still no single treatment dedicated for progressive MS. Finding a treatment that would result in diminished microglial cell activity in progressive MS would be an attractive way toward drug development for the progressive forms of the disease. MR imaging has been the gold standard for imaging MSrelated changes, but conventional MR imaging has a low detection threshold for diffuse white matter changes typical of progressive disease. Hence, other functional imaging methods, such as advanced MR imaging methods and PET imaging using TSPO radioligands, are needed to complement conventional MR imaging.

Our work supports the notion that TSPO PET imaging, together with conventional and nonconventional MR imaging, could potentially be used as an imaging biomarker in, for example, proof-ofconcept treatment studies of progressive MS, for which reduced radioligand binding could be interpreted as a quantifiable sign of reduced microglial activation. Interestingly, an in vivo ${ }^{11} \mathrm{C}-\mathrm{PK} 11195$ study has already shown that glatiramer acetate treatment leads to reduced microglial activation in relapsing-remitting MS (33). Future studies will have to elucidate the prognostic significance of increased microglial activation in the normal-appearing white matter and the implications of being able to therapeutically reduce microglial activation.

The utility of the focal DTH EAE model to demonstrate both novel diagnostic techniques and treatment effects has been demonstrated in earlier studies $(23,34)$. The advantage of using a focal model over the more commonly used disseminated models is that it is possible to monitor a specific lesion without the interference from new or neighboring lesions. Noninterference is especially important in micro-PET experiments because of the inherent low spatial resolution of the resultant image (35). The previous studies focused on the prevention of new lesion formation within the CNS because the treatment was already started simultaneously when the lesion was activated peripherally (23). In the present study, we examined the effects of fingolimod on a well-established DTH EAE lesion, which is a more clinically relevant paradigm, also in terms of progressive MS. The reduction of microglial activation surrounding the lesion occurred relatively quickly, after only $28 \mathrm{~d}$ of treatment, highlighting the efficacy of fingolimod in this model. This reduction is probably due to the direct effects of fingolimod within the CNS rather than the depletion of T cells at this short time point.

\section{CONCLUSION}

This study demonstrates the effectiveness of ${ }^{18} \mathrm{~F}-\mathrm{GE} 180$ as a second-generation TSPO ligand in detecting the treatment effect of fingolimod in reducing microglial activation in a chronic focal rodent model of MS. The ability to visualize the reduction of microglia in vivo using PET imaging potentially provides a key tool in following this surrogate marker for diffuse inflammation in progressive MS.

\section{DISCLOSURE}

The costs of publication of this article were defrayed in part by the payment of page charges. Therefore, and solely to indicate this fact, this article is hereby marked "advertisement" in accordance with 18 USC section 1734 . This study was financially supported by the Finnish Academy, Biocentre Finland, GE Healthcare, Ltd., and the European Union's Seventh Framework Programme (FP7/ 2007-2013) under grant agreement HEALTH-F2-2011-278850 (INMiND). No other potential conflict of interest relevant to this article was reported.

\section{ACKNOWLEDGMENTS}

We thank Professor David Leppert for fruitful discussions. The staff participating in executing the studies at the Turku PET Centre are gratefully acknowledged.

\section{REFERENCES}

1. Sormani MP, Bruzzi P. MRI lesions as a surrogate for relapses in multiple sclerosis: a meta-analysis of randomised trials. Lancet Neurol. 2013;12:669676.

2. Confavreux C, Vukusic S, Moreau T, Adeleine P. Relapses and progression of disability in multiple sclerosis. N Engl J Med. 2000;343:1430-1438.

3. Howell OW, Rundle JL, Garg A, Komada M, Brophy PJ. Reynolds R. Activated microglia mediate axoglial disruption that contributes to axonal injury in multiple sclerosis. J Neuropathol Exp Neurol. 2010;69:1017-1033.

4. Barkhof F. The clinico-radiological paradox in multiple sclerosis revisited. Curr Opin Neurol. 2002;15:239-245.

5. Papadopoulos V, Baraldi M, Guilarte TR, et al. Translocator protein (18kDa): new nomenclature for the peripheral-type benzodiazepine receptor based on its structure and molecular function. Trends Pharmacol Sci. 2006;27:402-409.

6. Chen MK, Baidoo K, Verina T, Guilarte TR. Peripheral benzodiazepine receptor imaging in CNS demyelination: functional implications of anatomical and cellular localization. Brain. 2004;127:1379-1392.

7. Politis M, Giannetti P, Su P, et al. Increased PK11195 PET binding in the cortex of patients with MS correlates with disability. Neurology. 2012;79:523-530. 
8. Banati RB. Visualising microglial activation in vivo. Glia. 2002;40:206-217.

9. Rissanen E, Tuisku J, Rokka J, et al. In vivo detection of diffuse inflammation in secondary progressive multiple sclerosis using PET imaging and the radioligand ${ }^{11}$ C-PK11195. J Nucl Med. 2014;55:939-944.

10. Ching AS, Kuhnast B, Damont A, Roeda D, Tavitian B, Dolle F. Current paradigm of the $18-\mathrm{kDa}$ translocator protein (TSPO) as a molecular target for PET imaging in neuroinflammation and neurodegenerative diseases. Insights Imaging. 2012;3:111-119.

11. Dickens AM, Vainio S, Marjamaki P, et al. Detection of microglial activation in an acute model of neuroinflammation using PET and radiotracers ${ }^{11} \mathrm{C}$-(R)PK11195 and ${ }^{18}$ F-GE-180. J Nucl Med. 2014;55:466-472.

12. Wickstrøm T, Clarke A, Gausemel I, et al. The development of an automated and GMP compliant FASTlab ${ }^{\text {TM }}$ synthesis of $\left[{ }^{18} \mathrm{~F}\right] \mathrm{GE}-180$; a radiotracer for imaging translocator protein (TSPO). J Labelled Comp Radiopharm. 2014;57:42-48.

13. Jeffery DR. Recent advances in treating multiple sclerosis: efficacy, risks and place in therapy. Ther Adv Chronic Dis. 2013;4:45-51.

14. Brinkmann V, Davis MD, Heise CE, et al. The immune modulator FTY720 targets sphingosine 1-phosphate receptors. J Biol Chem. 2002;277:21453-21457.

15. Cohen JA, Chun J. Mechanisms of fingolimod's efficacy and adverse effects in multiple sclerosis. Ann Neurol. 2011;69:759-777.

16. Foster CA, Howard LM, Schweitzer A, et al. Brain penetration of the oral immunomodulatory drug FTY720 and its phosphorylation in the central nervous system during experimental autoimmune encephalomyelitis: consequences for mode of action in multiple sclerosis. J Pharmacol Exp Ther. 2007;323:469-475.

17. Coelho RP, Payne SG, Bittman R, Spiegel S, Sato-Bigbee C. The immunomodulator FTY720 has a direct cytoprotective effect in oligodendrocyte progenitors. J Pharmacol Exp Ther. 2007;323:626-635.

18. Serres S, Anthony DC, Jiang Y, et al. Comparison of MRI signatures in pattern I and II multiple sclerosis models. NMR Biomed. 2009;22:1014-1024.

19. Lucchinetti C, Bruck W, Parisi J, Scheithauer B, Rodriguez M, Lassmann H. Heterogeneity of multiple sclerosis lesions: implications for the pathogenesis of demyelination. Ann Neurol. 2000;47:707-717.

20. Anthony DC, Miller KM, Fearn S, et al. Matrix metalloproteinase expression in an experimentally-induced DTH model of multiple sclerosis in the rat CNS. J Neuroimmunol. 1998;87:62-72.

21. Caporale V, Alessandrini B, Villa P, Del Papa S. Global perspectives on animal welfare: Europe. Rev Sci Tech. 2005;24:567-577.
22. Matyszak MK, Perry VH. Demyelination in the central nervous system following a delayed-type hypersensitivity response to bacillus Calmette-Guérin. Neuroscience. 1995;64:967-977.

23. Anthony DC, Sibson NR, Losey P, Meier DP, Leppert D. Investigation of immune and CNS-mediated effects of fingolimod in the focal delayed-type hypersensitivity multiple sclerosis model. Neuropharmacology. 2014;79:534-541.

24. McLean IW, Nakane PK. Periodate-lysine-paraformaldehyde fixative: a new fixation for immunoelectron microscopy. J Histochem Cytochem. 1974;22:10771083.

25. Farde L, Eriksson L, Blomqvist G, Halldin C. Kinetic analysis of central ${ }^{11} \mathrm{C}$ raclopride binding to D2-dopamine receptors studied by PET: a comparison to the equilibrium analysis. J Cereb Blood Flow Metab. 1989;9:696-708.

26. Cosenza-Nashat M, Zhao ML, Suh HS, et al. Expression of the translocator protein of $18 \mathrm{kDa}$ by microglia, macrophages and astrocytes based on immunohistochemical localization in abnormal human brain. Neuropathol Appl Neurobiol. 2009;35:306-328.

27. Cohen JA, Barkhof F, Comi G, et al. Oral fingolimod or intramuscular interferon for relapsing multiple sclerosis. N Engl J Med. 2010;362:402-415.

28. Mehling M, Kappos L, Derfuss T. Fingolimod for multiple sclerosis: mechanism of action, clinical outcomes, and future directions. Curr Neurol Neurosci Rep. 2011;11:492-497.

29. Groves A, Kihara Y, Chun J. Fingolimod: direct CNS effects of sphingosine 1phosphate $(\mathrm{S} 1 \mathrm{P})$ receptor modulation and implications in multiple sclerosis therapy. J Neurol Sci. 2013;328:9-18.

30. Frischer JM, Bramow S, Dal-Bianco A, et al. The relation between inflammation and neurodegeneration in multiple sclerosis brains. Brain. 2009;132:1175-1189.

31. Prineas JW, Kwon EE, Cho ES, et al. Immunopathology of secondary-progressive multiple sclerosis. Ann Neurol. 2001;50:646-657.

32. Magliozzi R, Howell OW, Reeves C, et al. A gradient of neuronal loss and meningeal inflammation in multiple sclerosis. Ann Neurol. 2010;68:477-493.

33. Ratchford JN, Endres CJ, Hammoud DA, et al. Decreased microglial activation in MS patients treated with glatiramer acetate. J Neurol. 2012;259:1199-1205.

34. Serres S, Anthony DC, Jiang Y, et al. Systemic inflammatory response reactivates immune-mediated lesions in rat brain. J Neurosci. 2009;29:4820-4828.

35. Levin CS, Hoffman EJ. Calculation of positron range and its effect on the fundamental limit of positron emission tomography system spatial resolution. Phys Med Biol. 1999;44:781-799. 